\title{
BMJ Open Tuberculosis care cascade in Zambia - identifying the gaps in order to improve outcomes: a population-based analysis
}

Patrick Lungu (D) , ${ }^{1,2}$ Andrew D Kerkhoff (D) , ${ }^{3}$ Clara C Kasapo, ${ }^{1}$ Judith Mzyece, ${ }^{1}$ Sulani Nyimbili, ${ }^{1}$ Rhehab Chimzizi, ${ }^{1}$ Andrew Silumesii, ${ }^{4}$ Mary Kagujje, ${ }^{5}$ Ramnath Subbaraman (D) , ${ }^{6}$ Monde Muyoyeta, ${ }^{5}$ Kennedy Malama ${ }^{7}$

To cite: Lungu $P$, Kerkhoff AD, Kasapo CC, et al. Tuberculosis care cascade in Zambia - identifying the gaps in order to improve outcomes: a populationbased analysis. BMJ Open 2021;11:e044867. doi:10.1136/ bmjopen-2020-044867

- Prepublication history and additional supplemental material for this paper are available online. To view these files, please visit the journal online. (http://dx.doi.org/10.1136/ bmjopen-2020-044867).

$\mathrm{PL}$ and $\mathrm{ADK}$ contributed equally.

Received 16 September 2020 Accepted 16 July 2021

Check for updates

(c) Author(s) (or their employer(s)) 2021. Re-use permitted under CC BY-NC. No commercial re-use. See rights and permissions. Published by BMJ.

For numbered affiliations see end of article.

Correspondence to Dr Patrick Lungu; patrickpj456@yahoo.co.uk

\section{ABSTRACT}

Objective Tuberculosis (TB) remains a leading cause of morbidity and mortality in Zambia, especially for people living with HIV (PLHIV). We undertook a care cascade analysis to quantify gaps in care and align programme improvement measures with areas of need.

Design Retrospective, population-based analysis. Setting We derived national-level estimates for each step of the TB care cascade in Zambia. Estimates were informed by WHO incidence estimates, nationally aggregated laboratory and notification registers, and individual-level programme data from four provinces. Participants Participants included all individuals with active TB disease in Zambia in 2018. We characterised the overall TB cascade and disaggregated by drug susceptibility results and HIV status.

Results In 2018, the total burden of TB in Zambia was estimated to be 72495 (range, 40 495-111 495) cases. Of these, 43387 (59.8\%) accessed TB testing, 40176 (55.4\%) were diagnosed with TB, 36431 (50.3\%) were started on treatment and 32700 (45.1\%) completed treatment. Among all persons with TB lost at any step along the care cascade ( $n=39795), 29108$ (73.1\%) were lost prior to accessing diagnostic services, 3211 (8.1\%) prior to diagnosis, 3745 (9.4\%) prior to initiating treatment and 3731 (9.4\%) prior to treatment completion. PLHIV were less likely than HIV-negative individuals to successfully complete the care cascade ( $42.8 \%$ vs $50.2 \%$, $\mathrm{p}<0.001)$. Among those with rifampicin-resistant TB, there was substantial attrition at each step of the cascade and only $22.8 \%$ were estimated to have successfully completed treatment.

Conclusions Losses throughout the care cascade resulted in a large proportion of individuals with TB not completing treatment. Ongoing health systems strengthening and patient-centred engagement strategies are needed at every step of the care cascade; however, scale-up of active case finding strategies is particularly critical to ensure individuals with TB in the population reach initial stages of care. Additionally, a renewed focus on PLHIV and individuals with drug-resistant TB is urgently needed to improve TB-related outcomes in Zambia.

\section{BACKGROUND}

The WHO End TB Strategy aims to reduce tuberculosis (TB) incidence by $90 \%$ and

\section{Strengths and limitations of this study}

- The national tuberculosis (TB) care cascade for Zambia in 2018 was characterised in order to identify gaps in care.

- The TB care cascade was constructed for all patients with TB as well as according to drug susceptibility results and HIV status.

- The analysis was informed by a published set of methodologies and used several data sources to derive estimates.

- Enhanced TB surveillance programmes, including the use of unique TB patient identifiers, would allow for real-time monitoring and improved estimates to inform programmatic strengthening.

TB-related deaths by $95 \%$ between 2015 and 2035. ${ }^{1}$ While many high-burden countries in sub-Saharan Africa, including Zambia, have demonstrated large reductions in new TB cases and associated mortality, there remains significant need for improved TB care delivery. ${ }^{2} \mathrm{~TB}$ remains a leading cause of morbidity and mortality in Zambia, especially among people living with HIV (PLHIV) ${ }^{23}$ In 2019, there were approximately 59000 new individuals with active TB disease in Zambia (incidence rate of 333 per 100000 per year), which resulted in 15400 TB-related deaths, of which $62 \%$ were among PLHIV. ${ }^{2}$ Despite substantial declines in TB incidence over the last decade, Zambia still has the seventh highest TB incidence in sub-Saharan Africa and remains one of $30 \mathrm{WHO}$ high TB burden priority countries. ${ }^{2}$

The HIV 'cascade of care' is a public health model that outlines the key engagement steps required for PLHIV to ultimately achieve an undetectable viral load. This model has been widely applied by HIV programmes globally to inform and strengthen HIV care and delivery, and ultimately significantly increase the number of PLHIV who know their HIV 
status, are started on antiretroviral therapy (ART) and have suppressed viral loads. ${ }^{4}$ Similarly, a national TB care cascade can provide key insights to identify and quantify gaps in the diagnosis and care of patients with TB that could then help guide programmatic and research priorities by aligning limited resources with the areas of greatest need. ${ }^{56}$ However, to date, only three high-burden TB countries-South Africa, India and Madagascar-have undertaken and published national-level TB care cascade analyses. $^{7-9}$

We sought to construct a national TB cascade of care for Zambia to evaluate care delivery for individuals with active TB disease through enumeration of gaps in the overall care cascade in 2018 as well as disaggregated by rifampicin susceptibility results and HIV status. Estimates were derived using multiple data sources, and the overall approach was informed by a recently published methodology for constructing TB care cascades. ${ }^{6}$

\section{METHODS}

\section{Study design}

We undertook a retrospective, population-based study to characterise the TB care cascade in Zambia in 2018. All Zambians estimated to be living with TB in 2018 were included in the analysis, regardless of age, HIV status, diagnosis status (ie, diagnosed or undiagnosed TB), TB drug susceptibility status or TB type (ie, new or retreatment).

\section{Setting}

Zambia has an estimated population of 18400000 people. ${ }^{10}$ It has a high prevalence of HIV (11.5\% among adults aged 15-49 years old), and it is estimated that at least 1.2 million persons are living with HIV. ${ }^{11} \mathrm{~TB}$ is a major public health problem in Zambia; ${ }^{3}$ during the last national TB prevalence survey conducted in 2013 and 2014 , the prevalence of microbiologically confirmed TB was estimated to be 638 per 100000 persons and was five times higher among HIV-positive individuals compared with HIV-negative individuals. ${ }^{12}$

Testing and treatment for TB are almost universally provided within Zambia's public health system. While exact estimates are not available, likely $<1 \%$ of all individuals with $\mathrm{TB}$ are detected and managed within Zambia's private sector and the large majority are reported to Zambia's National TB Program (NTP) - this assumption is informed by a national data quality assessment conducted in $2019 .{ }^{13}$ Within the public health sector, the direct costs of all TB diagnostics and treatment are provided free of charge. In 2018, Xpert MTB/RIF was the recommended first-line diagnostic for all individuals undergoing evaluation for possible TB (pulmonary or extrapulmonary) in Zambia, as well as initial drug susceptibility testing (DST) $;{ }^{14}$ however, it was not universally available at all facilities, in which case routine TB investigations included acid fast bacilli (AFB) fluorescence or Ziehl-Neelsen microscopy and chest radiography, where available. Among those with confirmed rifampicin-resistant (RR) or multidrug-resistant (MDR) TB, it was recommended that either liquid culture or a molecular line probe assay should be used as follow-on tests for further DST. ${ }^{14}$ First-line TB treatment was provided to all patients without evidence of rifampicin resistance and consisted of isoniazid, rifampicin, ethambutol and pyrazinamide for 6-9 months in conformity with WHO recommendations. ${ }^{15}$ In 2018, Zambia began scaling up shorter treatment regimens that comprised new and repurposed TB drugs for 9-12 months for eligible patients with RR-TB and MDR-TBthis accounted for the majority of patients; ${ }^{16}{ }^{17}$ however, some patients still received longer MDR-TB treatment regimens that comprised several TB drugs, including an injectable agent, for at least 20 months.

In Zambia, patients diagnosed with $\mathrm{TB}$ are notified in a paper-based register and initiated on TB therapy at the corresponding TB treatment facility, which is also responsible for documentation of the treatment outcome of the patient. Data on diagnostic outcomes (laboratory register), notifications and treatment outcomes (notification register) are aggregated from each facility through the district office to the provincial level and then to the national level on a monthly basis.

\section{TB cascade data sources}

Several data sources were used to inform estimates within each of the five steps of the care cascade (table 1 , online supplemental appendix). To inform estimates of the overall burden of TB in Zambia in 2018 (step 1), WHO estimates of TB incidence from 2018 and 2017 were used. ${ }^{18-21}$ The proportion of total individuals with TB estimated to be RR was derived using estimates from the most recent national survey of TB drug resistance in Zambia; ${ }^{22}$ this source was chosen in order to ground estimates of RR-TB in empirical data. However, higher-end estimates from the latest Zambian national survey of TB drug resistance in 2008 were used to more closely align with WHO incidence estimates for RR-TB in 2018. Diagnostic outcomes (steps 2 and 3) were informed by a nationally aggregated database of TB diagnostics from 2018, which includes the number and type of investigations (Xpert or smear microscopy) and the number of patients with TB detected according to type of TB investigation and HIV status. All treatment outcomes (steps 4 and 5) were informed by a nationally aggregated TB treatment register from 2018.

Individual-level programmatic data from four Zambian provinces (Eastern, Lusaka, Southern, Western) regarding all patients investigated for $\mathrm{TB}$ and those started on treatment between 1 January and 31 December 2017 ( $\mathrm{n}=43896$ and $\mathrm{n}=11$ 814, respectively) were used to determine (1) the proportion of patients who had both positive Xpert and smear microscopy results, as well as (2) the proportion of patients who were Xpert-negative or smear-negative but received empirical TB therapy. This helped to further refine estimates for steps 2 and 3 by accounting for and removing duplicate patients (online supplemental appendix). Patient-level data were 
Table 1 Approach to and data sources for estimating each step of the tuberculosis care cascade in Zambia in 2018

\begin{tabular}{|c|c|c|c|c|c|}
\hline & $\begin{array}{l}\text { Step 1: TB } \\
\text { burden }\end{array}$ & Step 2: accessed tests & Step 3: diagnosed & $\begin{array}{l}\text { Step 4: notified } \\
\text { and treated }\end{array}$ & $\begin{array}{l}\text { successfully } \\
\text { treated }\end{array}$ \\
\hline All TB cases & $\begin{array}{l}\text { WHO estimates } \\
\text { of TB incidence } \\
\text { in } 2018 \text { plus } 50 \% \\
\text { of the number of } \\
\text { undetected cases } \\
\text { from } 2017 . .^{191}\end{array}$ & $\begin{array}{l}\text { Add the number of missed } \\
\text { cases to the total number } \\
\text { of DS-TB cases diagnosed } \\
\text { (step 3). } \\
\text { Missed cases estimated } \\
\text { based on TB test } \\
\text { sensitivity by HIV status } \\
\text { (informed by published } \\
\text { reports }{ }^{25-27} \text { ), corrected for } \\
\text { the number of patients } \\
\text { with negative TB tests } \\
\text { who were empirically } \\
\text { treated (informed by } \\
\text { unpublished individual- } \\
\text { level data from } 4 \text { Zambian } \\
\text { provinces in } 2017 \text { ). }\end{array}$ & $\begin{array}{l}\text { Back-calculated from the } \\
\text { number of cases notified } \\
\text { (step 4) and the proportion } \\
\text { of patients LTFU prior to } \\
\text { initiation of TB therapy. } \\
\text { Pretreatment LTFU } \\
\text { estimated based } \\
\text { on the difference } \\
\text { between the number } \\
\text { of microbiologically } \\
\text { confirmed DS-PTB cases } \\
\text { detected (informed by } \\
\text { aggregated facility-level } \\
\text { TB laboratory data from } \\
2018 \text { (unpublished)) } \\
\text { and the number of } \\
\text { microbiologically } \\
\text { confirmed DS-PTB cases } \\
\text { notified (informed by } \\
\text { aggregated facility-level } \\
\text { TB notification data from } \\
2018 \text { (unpublished)). }\end{array}$ & $\begin{array}{l}\text { Exact value } \\
\text { from aggregated } \\
\text { facility-level } \\
\text { TB notification } \\
\text { data from } 2018 \\
\text { (unpublished). }\end{array}$ & $\begin{array}{l}\text { Add DS-TB and } \\
\text { RR-TB cases } \\
\text { successfully } \\
\text { treated. }\end{array}$ \\
\hline $\begin{array}{l}\text { Rifampicin- } \\
\text { resistant TB } \\
\text { cases }\end{array}$ & $\begin{array}{l}\text { Overall TB } \\
\text { burden multiplied } \\
\text { by estimated } \\
\text { proportion of } \\
\text { cases with } \\
\text { rifampicin } \\
\text { resistance } \\
\text { (informed by most } \\
\text { recent Zambia } \\
\text { national TB drug } \\
\text { resistance survey } \\
\text { in } 2008^{22} \text { ). }\end{array}$ & $\begin{array}{l}\text { Back-calculated from } \\
\text { RR-TB cases diagnosed } \\
\text { (step 3) on the basis of } \\
\text { cases bacteriologically } \\
\text { diagnosed, by test type } \\
\text { and test sensitivity } \\
\text { (informed by published }^{\text {reports }}{ }^{2528} \text { ). }\end{array}$ & $\begin{array}{l}\text { Exact value from } \\
\text { aggregated facility-level } \\
\text { TB laboratory data from } \\
2018 \text { (unpublished). }\end{array}$ & $\begin{array}{l}\text { Exact value } \\
\text { from aggregated } \\
\text { facility-level } \\
\text { TB notification } \\
\text { data from } 2018 \\
\text { (unpublished). }\end{array}$ & $\begin{array}{l}\text { Exact value } \\
\text { from aggregated } \\
\text { facility-level } \\
\text { TB treatment } \\
\text { outcomes data } \\
\text { from } 2018 \\
\text { (unpublished). }\end{array}$ \\
\hline
\end{tabular}




\section{Step 1: TB burden}

Drugsusceptible TB cases, HIV-positive individuals
WHO 2019 analysis of DSTB incidence in 2017 plus $50 \%$ of the number of undetected cases from 2018. ${ }^{1921}$

$\begin{array}{ll}\text { Drug- } & \text { Total number of } \\ \text { susceptible } & \text { DS-TB cases } \\ \text { TB cases, } & \text { minus number } \\ \text { HIV-negative } & \text { of DS-TB } \\ \text { individuals } & \text { cases among } \\ & \begin{array}{l}\text { HIV-positive } \\ \text { individuals. }\end{array}\end{array}$

\section{Step 2: accessed tests}

Add the number of missed cases of DS-TB among HIV-positive individuals to the total number of DS-TB cases diagnosed among HIV-positive individuals (step 3). Missed cases estimated based on TB test sensitivity in HIV-positive individuals, corrected for the number of patients with negative TB tests who were empirically treated. $^{2526}$

\section{Total number of DS-TB} cases who accessed TB tests minus the number of DS-TB cases who accessed TB tests among HIV-positive individuals.
Total number of DS-TB cases diagnosed minus the number of DS-TB cases diagnosed among HIV-positive individuals. (pretreatment LTFU assumed to be the same independent of HIV status).

Back-calculated from notified (step 4) and the proportion of patients LTFU prior to initiation of

diagnosed minus
diagnosed among
sitive individuals.

\section{Step 5: \\ Step 4: notified successfully and treated treated}

Exact value Exact value from aggregated from aggregated facility-level facility-level TB notification TB treatment data from 2018 outcomes adjusted for the data from proportion of 2018 (number patients without successfully an HIV test. treated) adjusted (unpublished). for proportion of patients without an HIV test (unpublished).

$\begin{array}{ll}\text { Total number of } & \text { Total number of } \\ \text { DS-TB cases } & \text { DS-TB cases } \\ \text { notified minus } & \text { successfully } \\ \text { the number } & \text { treated minus the } \\ \text { of DS-TB } & \text { number of DS- } \\ \text { cases among } & \text { TB cases among } \\ \text { HIV-positive } & \text { HIV-positive } \\ \text { individuals } & \text { individuals } \\ \text { notified. } & \begin{array}{l}\text { successfully } \\ \text { treated. }\end{array}\end{array}$

DS-PTB, drug-susceptible pulmonary tuberculosis; DS-TB, drug-susceptible tuberculosis; LTFU, lost-to-follow-up; RR-TB, rifampicinresistant tuberculosis; TB, tuberculosis.

only available from 4 out of 10 provinces; however, they account for nearly $60 \%$ of Zambia's national TB notifications, and the range of socioeconomic characteristics of individuals as well as their access to healthcare services are representative of the other six provinces. ${ }^{23}{ }^{24}$ Unfortunately, robust data from 2018 to inform these estimates were unavailable; thus, we used 2017 data because they were well characterised and temporally close to the year for which we sought to characterise the TB care cascade.

Diagnostic sensitivity estimates of $\mathrm{Xpert}^{25}$ and smear microscopy $^{2627}$ for detection of TB stratified according to HIV status, as well as Xpert, ${ }^{25}$ molecular line probe assays $^{28}$ and liquid culture ${ }^{29}$ for rifampicin resistance, were informed by previously published systematic reviews and meta-analyses.

\section{TB cascade estimation methods}

We calculated national-level estimates for each step of the TB care cascade in Zambia in 2018 (table 1, online supplemental appendix). This included the following: step 1: the total burden of active TB disease (individuals with prevalent TB in 2018); step 2: the total number of individuals with $\mathrm{TB}$ who accessed $\mathrm{TB}$ testing; step 3: the total number who were diagnosed with $\mathrm{TB}$; step 4: the total number who were notified and started on TB treatment; and step 5: the total number who successfully completed
TB treatment. Each step of the cascade and the overall TB care cascade were calculated among all patients and disaggregated according to rifampicin resistance results (RR-TB and drug-susceptible TB (DS-TB)) and, among those with DS-TB, by HIV status. There were insufficient data available to characterise the RR-TB care cascade disaggregated according to HIV status. RR-TB was defined as the detection of rifampicin resistance on any clinical specimen using Xpert, molecular line probe assay or liquid culture; this definition therefore encompassed all patients with MDR-TB and extensively drug-resistant TB. DS-TB was defined as any TB case without known rifampicin resistance; thus, there is a possibility that patients with other forms of drug resistance, including isoniazid monoresistance, may have been included in this definition. However, unless rifampicin resistance is detected, TB DST is not routinely performed in Zambia-this reflects the clinical reality of many high-burden TB settings and conforms with WHO recommendations.

The approach to all estimates followed the recommendations outlined in a published set of methods for constructing national-level TB care cascades. ${ }^{6}$ An overview of the approach used to calculate each step of the TB care cascade is summarised in table 1 and is described in brief below. However, a highly detailed summary of all 
assumptions, calculations, estimates and data sources is summarised in the online supplemental appendix.

We first started with step 4 (the total number of patients who were notified and started on TB treatment, including new, relapse, treatment after failure, treatment after loss-to-follow-up patients and other previously treated individuals ${ }^{30}$ ) and step 5 (the total number who successfully completed TB treatment), which were both directly informed by exact values from aggregated facility-level notification data. Step 3 (the total number who were diagnosed with TB) was then back-calculated from the number of individuals notified (step 4) and the proportion of patients who were estimated to have been lost-to-follow-up (LTFU) prior to initiation of TB therapy (pretreatment LTFU), which was informed by aggregated facility-level laboratory data. Step 2 (the total number of individuals with TB who accessed TB testing) was calculated by adding the number of individuals with TB who would not have been microbiologically diagnosed due to the incomplete sensitivity of TB diagnostic tests (based on published reports), corrected for the number of testnegative patients with $\mathrm{TB}$ who were empirically diagnosed, to the number of total patients with TB diagnosed (step 3). The overall approach for steps 2-5 was similar for both DS-TB and RR-TB (table 1, online supplemental appendix). The overall TB burden (all forms) was estimated using the WHO TB incidence estimate for 2018, plus $50 \%$ of the number of all individuals with $\mathrm{TB}$ who remained undiagnosed in 2017; a 50\% estimate has previously been used and assumed that the remaining $50 \%$ of undiagnosed individuals with TB in 2017 either self-cured or died. ${ }^{831}$ To determine the total number of individuals with rifampicin-resistant TB (step 1), we multiplied the overall TB burden by the proportion of all patients who had rifampicin resistance detected during the Zambian national drug resistance survey. ${ }^{22}$ The total number of individuals with DS-TB was calculated using the total TB burden minus the number of RR-TB cases.

All 'gaps' between each step were calculated by taking the difference in the total number of individuals with TB and the uncertainty estimate (either 95\% CI or range) between the succeeding and proceeding steps. All TB care cascades were depicted graphically using bar charts representing the absolute number of cases and the associated uncertainty measurement (if applicable). For each step of each cascade, proportions relative to the total TB burden (step 1) as well as relative to the prior step were calculated. It should be noted that several steps of the cascade used exact numbers from aggregated facility-level programmatic data (steps 3, 4 and 5). For the purposes of these analyses, data were assumed to be accurate and complete; however, such data may be incompletely recorded and a small proportion may be entered incorrectly-estimates of uncertainty around exact values from programmatic data were unavailable. Furthermore, unique patient identifiers are not available within Zambia's NTP and thus this analysis does not present a cohort of individuals who were tracked through each step of the TB care cascade.
While we assumed for the purposes of this analysis that the same patients were being characterised at each step of the cascade, one cannot exclude the possibility that different individuals are being captured at different steps of the care cascade.

\section{Evaluating diagnostic and treatment outcomes}

To understand any progress that may have underpinned the 2018 TB care cascade, we also evaluated TB diagnostic and treatment completion trends from 2015 to 2018. Using facility-level aggregated laboratory data, we plotted (1) the total number of sputum Xpert tests undertaken each year against the total number of pulmonary TB cases diagnosed each year, including the proportion that was microbiologically confirmed, as well as (2) the total number of Xpert tests undertaken (on any specimen) each year against the total number of RR-TB cases diagnosed and notified each year. We also plotted the proportion (and corresponding 95\% CI) of patients with TB each year who started TB treatment who successfully completed it, disaggregated according to TB type: (1) new/relapse pulmonary TB-overall, (2) HIV-positive new/relapse pulmonary TB, (3) HIV-negative new/ relapse pulmonary $\mathrm{TB},(4)$ retreatment $\mathrm{TB}$ not including individuals who experienced relapse, and (5) extrapulmonary TB.

\section{RESULTS}

\section{Overall national TB care cascade for $\mathbf{2 0 1 8}$}

In 2018, the overall burden of TB in Zambia was estimated to comprise 72495 individuals with TB (range, 40 495-111 495; table 2, figure 1A). Of the total burden of individuals with TB, 43387 (range, 42 390-44 710; 59.8\%) were estimated to have sought care for their TB illness and undergone microbiological TB testing. Among these individuals, 40176 (range, 40 128-40 212; proportion of total TB burden 55.4\%) were diagnosed with TB, 36431 (exact value; proportion of total TB burden $50.3 \%$ ) were notified and initiated on TB therapy, and 32700 (exact value; proportion of total TB burden $45.1 \%$ ) completed TB therapy. Therefore, 39795 (range, 8191-79 191; $54.9 \%$ ) of the estimated individuals with TB in 2018 did not complete the care cascade (table 3). Individuals who did not seek care for their TB illness or who sought care but did not undergo microbiological TB testing accounted for 29108 (range, 0-66 777; 73.1\%) individuals with TB lost along the cascade in 2018 (table 3). Suboptimal empirical diagnosis of individuals with TB who had negative microbiological test results (due to incomplete diagnostic sensitivity of these tests) contributed to an additional 3211 (95\% CI 2262 to $4506 ; 8.1 \%$ ) missed TB cases; losses to follow-up prior to TB treatment initiation accounted for 3745 (95\% CI 3697 to $3781 ; 9.4 \%$ ) patients lost, and unfavourable outcomes (loss-to-follow-up, death and treatment failure) prior to $\mathrm{TB}$ treatment completion accounted for 3731 (exact value; 9.4\%) patients lost. 


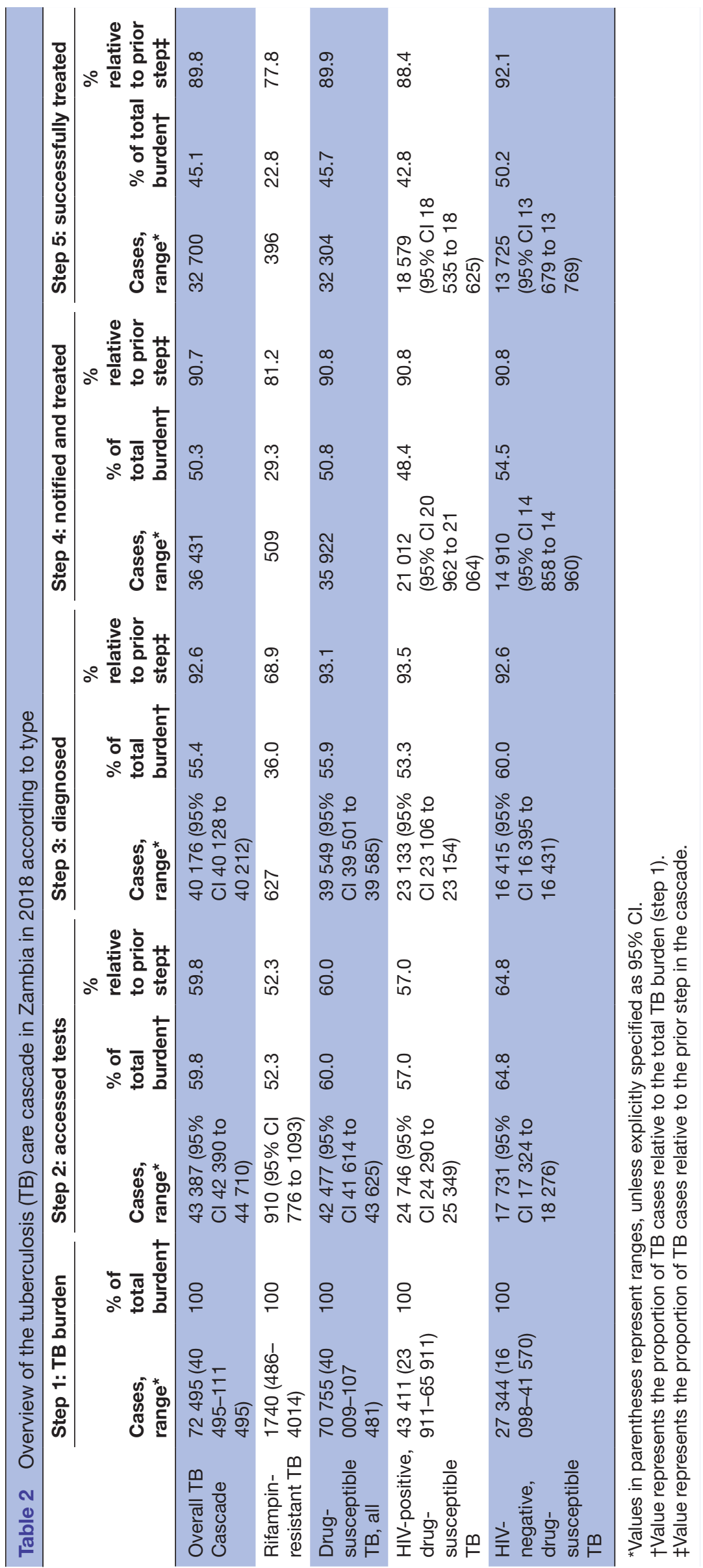




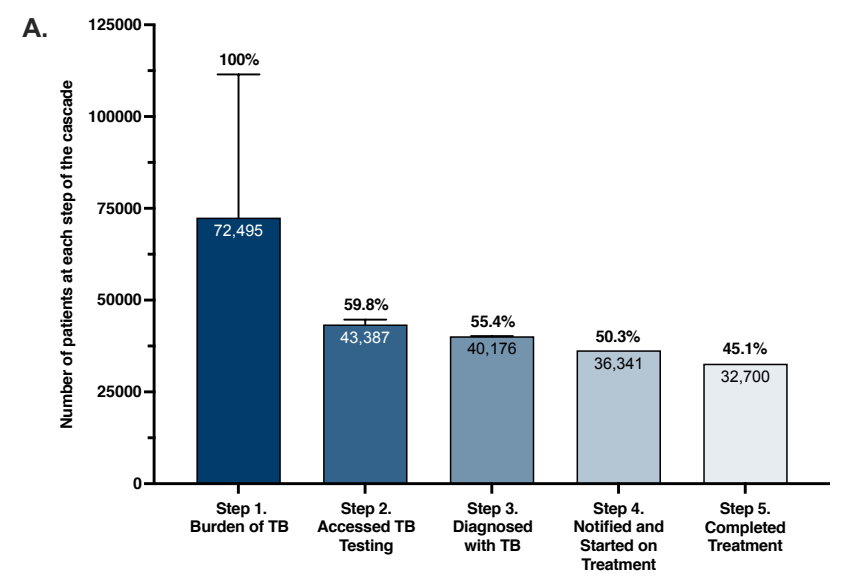

B.

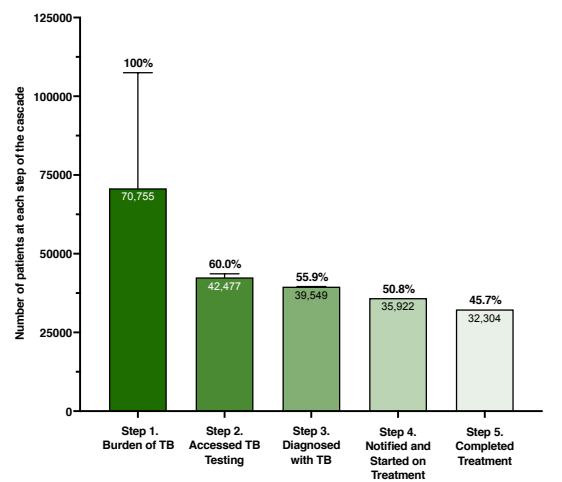

D.

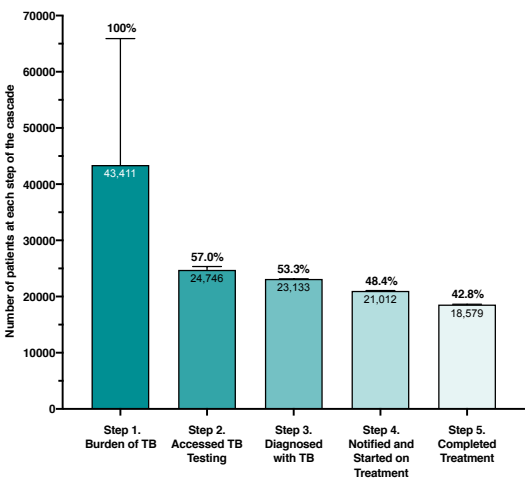

C.

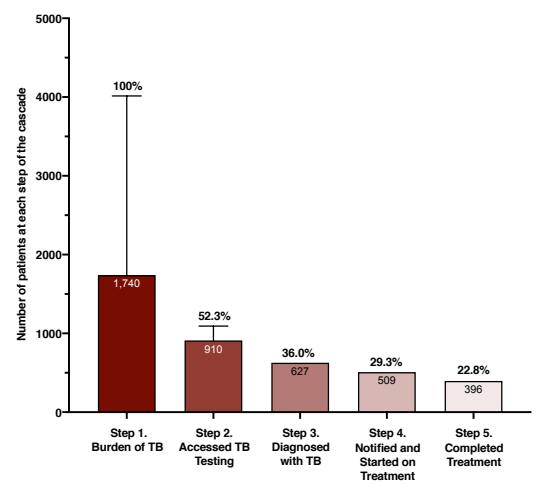

E.

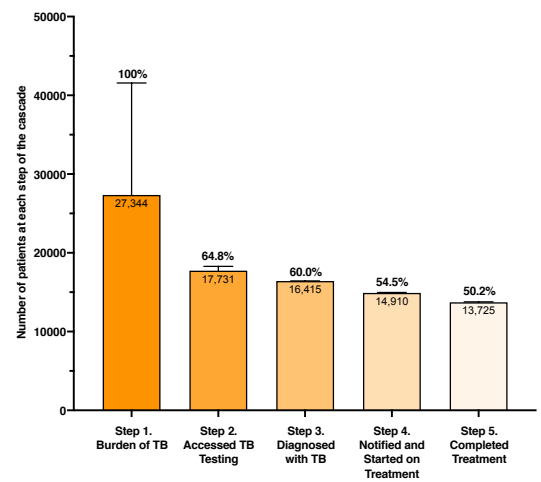

Figure 1 The tuberculosis (TB) care cascade in Zambia in 2018 among (A) all tuberculosis cases; (B) drug-susceptible cases; (C) rifampicin-resistant cases; (D) drug-susceptible cases among HIV-positive individuals; and (E) drug-susceptible cases among HIV-negative individuals.

\section{TB care cascade by drug susceptibility results}

We estimated the burden of individuals with DS-TB in 2018 to be 70755 (range, 40 009-107 481), approximately $97.6 \%$ of the total TB burden. The DS-TB cascade was largely similar to the overall TB cascade, with 32304 (exact value; $45.7 \%$ ) of all individuals being diagnosed with $\mathrm{TB}$, initiating on and completing $\mathrm{TB}$ treatment (table 2, figure 1B). The total number of RR-TB cases was estimated to be 1740 (range, 486-4014), or $2.4 \%$ of the total TB burden. Compared with individuals with DS-TB, individuals with RR-TB were substantially less likely to access microbiological TB testing $(52.3 \%$ vs $60.0 \%$, $\mathrm{p}<0.001)$, have their TB diagnosed $(68.9 \%$ vs $93.1 \%$, $\mathrm{p}<0.001)$, be notified and initiated on $\mathrm{TB}$ treatment $(81.2 \%$ vs $90.8 \%, \mathrm{p}<0.001)$ and to complete TB therapy $(77.8 \%$ vs $89.9 \%, \mathrm{p}<0.001)$ (figure $1 \mathrm{C})$. Thus, only 396 (exact value; $22.1 \%$ ) individuals with RR-TB completed the TB care cascade. The majority of those with RR-TB along the pathways were due to individuals who did not seek care or who did not have access to TB and/or DST, accounting for 830 cases (range, $0-2961 ; 61.7 \%$ ) (table 3); however, 283 (95\% CI 149 to 466; 21.1\%) of lost RR-TB cases were among those who accessed TB testing and had RR-TB missed, 118 (exact value; 8.8\%) were among those who had RR-TB detected but were not notified and started on appropriate TB therapy, and 113 (exact value; 


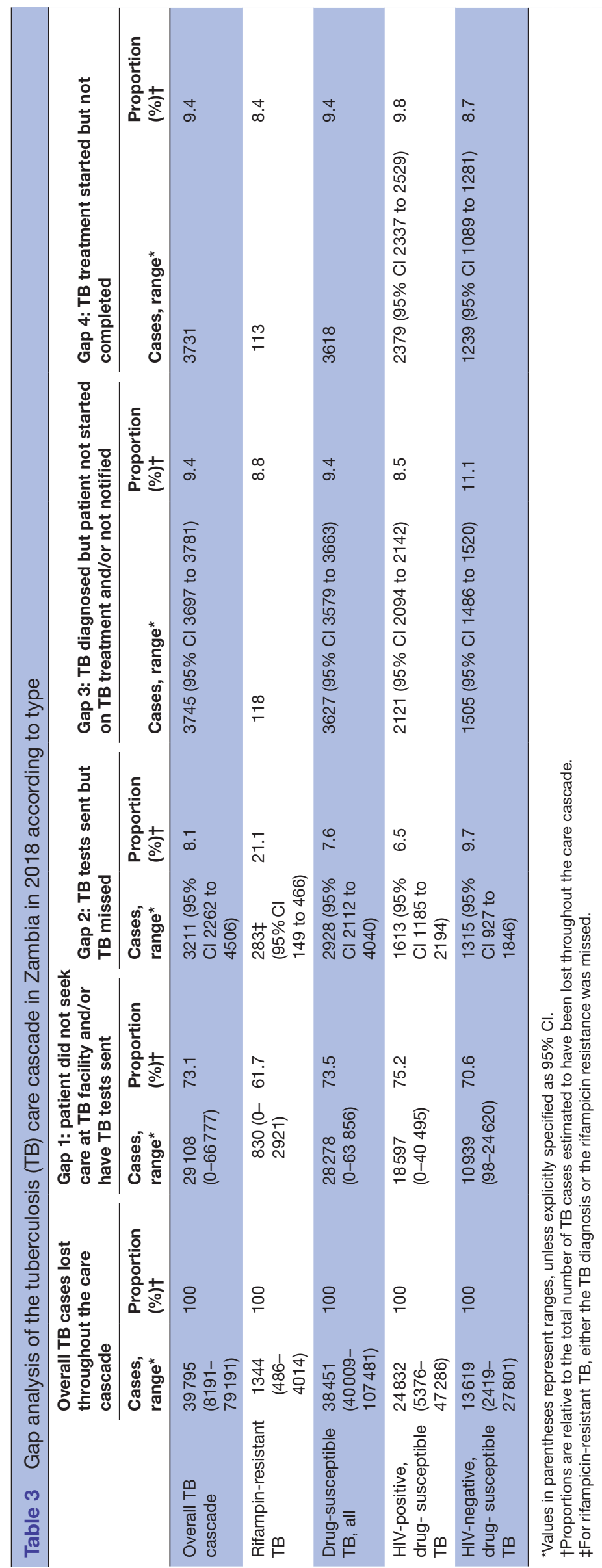

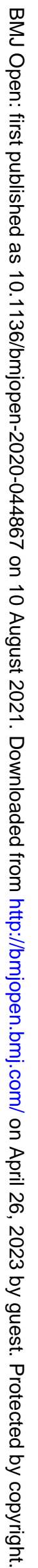


A.

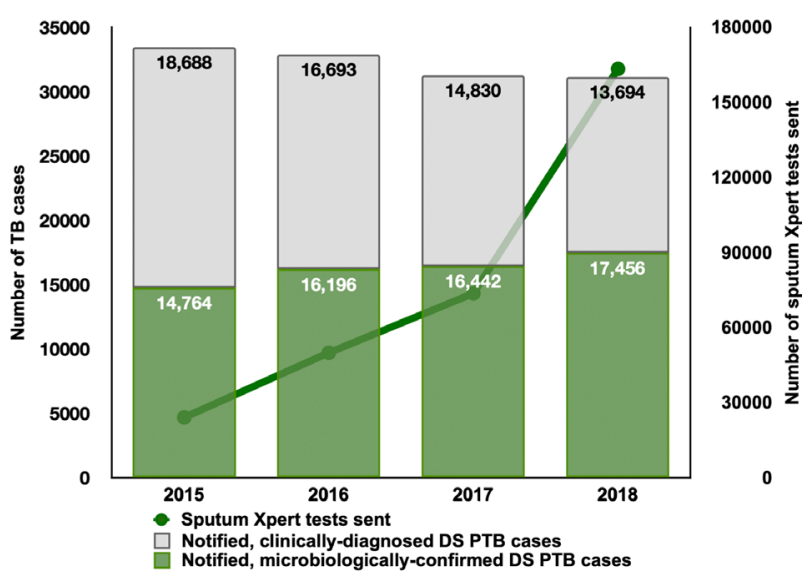

B.

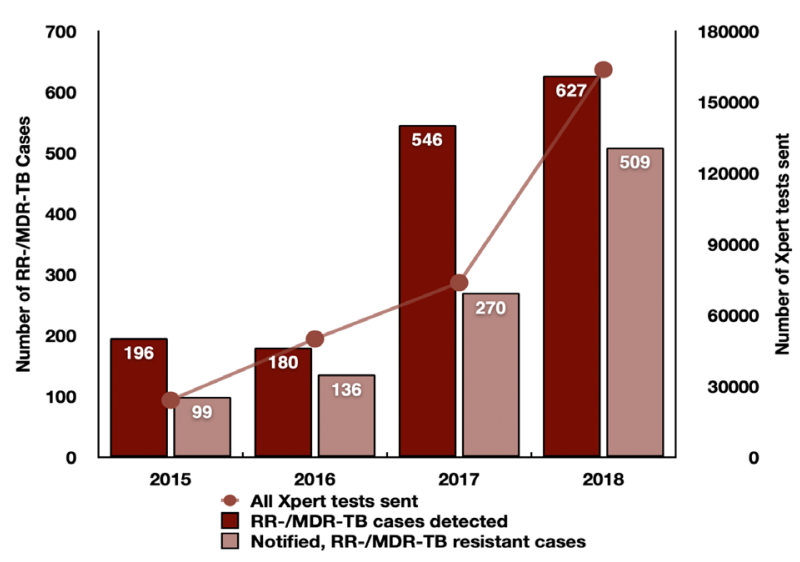

Figure 2 Diagnoses and notifications of (A) all forms of drug-susceptible pulmonary TB in Zambia between 2015 and 2018 and (B) drug-resistant TB in Zambia between 2015 and 2018. DS, drug susceptible MDR-TB, multidrug-resistant tuberculosis; PTB, pulmonary tuberculosis; RR-TB, rifampicin-resistant tuberculosis; TB, tuberculosis.

$8.4 \%)$ were among those who did not complete RR-TB therapy (table 3 ).

\section{DS-TB care cascade by HIV status}

Of 70755 individuals with DS-TB in 2018, 43411 (range, 23 911-65911; 61.4\%) were estimated to be among PLHIV. Compared with patients with DS-TB who were HIV-negative, HIV-positive patients with DS-TB were less likely to access microbiological TB testing (57.0 vs $64.8 \%$, $\mathrm{p}<0.001)$ and were less likely to complete TB treatment ( $88.4 \%$ vs $92.1 \%, \mathrm{p}<0.001)$. This resulted in a lower overall proportion of HIV-positive patients compared with HIVnegative patients completing the TB care cascade $(42.8 \%$ vs $50.2 \%, \mathrm{p}<0.001$; table 2 , figure $1 \mathrm{D}, \mathrm{E})$. For both HIVpositive and HIV-negative patients with DS-TB, the largest loss in the care cascade was due to patients not accessing microbiological TB testing, resulting in 18597 (range, 0-40 495; 75.2\%) and 10939 (range, 98-24 620; 70.6\%) missed patients, respectively (table 3 ).

\section{TB diagnosis trends from 2015 to 2018}

Between 2015 and 2018, Xpert MTB/RIF was increasingly used as the first-line TB diagnostic tool in Zambia, where 24140 Xpert tests were sent for suspected pulmonary TB in 2015, which increased to 163470 sent in 2018 (figure 2A). During this same period, the number of sputum AFB smear microscopy investigations decreased from 95300 in 2015 to 25323 in 2018 . While there was a small decrease in the absolute number of pulmonary TB cases diagnosed and notified in 2018 compared with 2015
(31 272 vs 33 452), the proportion of microbiologically confirmed TB cases that were notified during that period substantially increased $(56.0 \%$ (95\% CI 55.5 to 56.6$)$ vs $44.1 \%$ (95\% CI 43.6 to 44.7$)$; figure 2A). The scale-up of Xpert testing between 2015 and 2018 was also associated with a more than threefold increase in the annual number of RR cases detected (627 vs 196) and more than fivefold increase in the annual number of RR-TB cases that were notified and started on appropriate TB treatment (509 vs 99; figure 2B). During this period, there was a corresponding reduction in the proportion of RR-TB cases LTFU prior to the initiation of TB treatment, from $49.5 \%$ in 2015 to $18.8 \%$ in 2018 ( $\mathrm{p}<0.001)$.

\section{TB treatment completion trends from 2015 to 2018}

Finally, we examined trends in the proportion of patients with DS-TB who completed TB treatment once they were notified and initiated on therapy (figure 3). Among new/ relapse pulmonary $\mathrm{TB}$ cases, treatment completion rates steadily increased between 2015 and 2018 (86.2 (95\% CI 85.8 to 86.6 ) vs $90.3 \%$ (95\% CI 90.0 to 90.7$)$, $\mathrm{p}<0.001)$. There was also a trend towards improved TB treatment completion rates from 2015 to 2018 among retreatment pulmonary TB cases $(84.4 \%$ (95\% CI 83.3 to 85.5$)$ vs $87.2 \%$ (95\% CI 84.5 to 89.6), $\mathrm{p}=0.06$ ); however, completion rates declined from 2017 to 2018 (95.0\% (95\% CI 93.4 to 96.3 ) vs $87.2 \%$ (95\% CI 84.5 to 89.6$), \mathrm{p}<0.001)$. From 2015 to 2018, the proportion of patients with extrapulmonary TB completing TB treatment also improved $(80.3 \%$ (95\% CI 


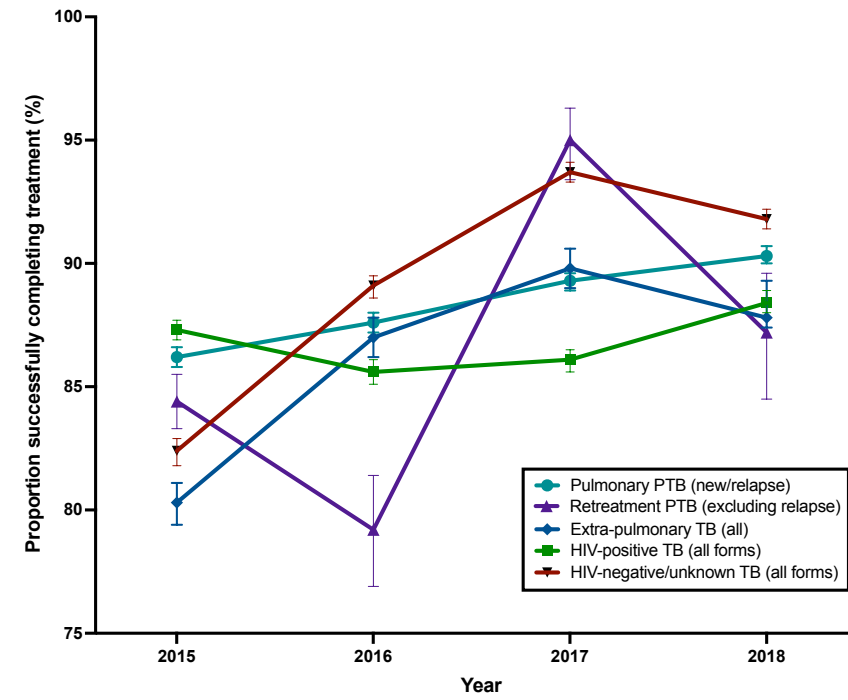

Figure 3 Overview of drug-susceptible tuberculosis (TB) treatment outcomes in Zambia between 2015 and 2018, disaggregated according to tuberculosis type. Shapes represent the proportion of patients completing tuberculosis treatment.PTB,pulmonary tuberculosis

79.4 to 81.1 ) vs $87.8 \%$ (95\% CI 87.4 to 89.3 ), $\mathrm{p}<0.001$ ). The proportion of HIV-positive patients completing TB therapy did not meaningfully change from 2015 to 2018 (87.3\% (95\% CI 86.9 to 87.7 ) vs $88.4 \%$ (95\% CI 88.0 to 88.9), $\mathrm{p}=0.001)$. Improvements in treatment completion rates from 2015 to 2018 were seen among patients who had a negative or unknown HIV status $(82.4 \%$ (95\% CI 81.8 to 82.9 ) vs $91.8 \%$ (95\% CI 91.4 to 92.2 ), $\mathrm{p}<0.001$ ), although there was a small decline between 2017 and 2018 (93.7\% (95\% CI 93.3 to 94.1 ) vs $91.8 \%$ (95\% CI 91.4 to 92.2$), \mathrm{p}<0.001$; figure 3 ). In 2018, a lower proportion of HIV-positive TB patients completed therapy compared with HIV-negative patients (difference $3.4 \% \quad(95 \%$ CI 2.8 to 4.0$), p<0.001$ ). Differences in the proportion of patients completing TB therapy according to HIV status were driven by a higher absolute number and proportion of cases who died or were LTFU during treatment among HIV-positive individuals compared with HIV-negative individuals (online supplemental table 1).

\section{DISCUSSION}

In this study we found that less than half of all TB cases in Zambia in 2018 were diagnosed with TB, initiated on TB treatment and completed therapy. We identified important losses at each step of the TB care cascade; however, we estimate that more than $40 \%$ of all individuals with TB in Zambia are not accessing microbiological TB testing-this accounted for nearly three-quarters of the estimated number of cases lost throughout the cascade. These results highlight important research and programmatic priorities for improving TB care and TB-related outcomes in Zambia.

This represents the fourth national TB care cascade that has been characterised from a high-burden TB country and builds on similar analyses from South Africa, India and Madagascar. ${ }^{7-9}$ Our overall TB care cascade results are similar to those from these countries, which each found that only about $50 \%$ of all patients with TB were progressing through all steps of the care cascade and completing TB treatment. In India the largest losses in the care cascade were among those who did not access TB testing (28\% of all cases) ${ }^{7}$ In Madagascar the largest losses in the cascade were among those who were not diagnosed with TB despite seeking care and accessing a TB diagnostic facility (26\% of all cases) ${ }^{9}$ while in South Africa steady losses were seen prior to TB diagnosis (12\% of all cases), prior to starting TB treatment (13\% of all cases) and prior to successful completion of TB therapy (17\% of all cases).$^{8}$ In Zambia, $40 \%$ were estimated to have not accessed TB testing, while $4 \%-5 \%$ of all TB cases were lost at each subsequent step of the care cascade. These differences highlight specific programmatic needs at different steps within the TB care cascade for each country and provides insight into the unique challenges that each faces.

Our results are consistent with several TB prevalence surveys suggesting that a large proportion of individuals with TB face barriers to healthcare-seeking, barriers to accessing microbiological TB testing or both. ${ }^{32} 33$ Unfortunately, we are not able to discern whether the estimated $40 \%$ gap in patients not accessing TB microbiological investigations is predominantly driven by (1) individuals who fundamentally lacked access to primary health and TB facilities, (2) individuals who either delayed or never presented to TB testing facilities for evaluation of their illness, or (3) individuals who sought care at health facilities but their illness was not suspected to be TB and thus they never had TB testing undertaken. ${ }^{34}$ After onset of symptoms, individuals with undiagnosed TB may have long and complex journeys to TB care as they often face many barriers to care-seeking and accessing TB services (eg, lack of knowledge, lack of social support, lack of time/finances, TB/HIV-related stigma, cultural and gender norms). ${ }^{33} 3536$ In the last Zambian national TB prevalence survey conducted in 2013 and 2014, only 60\% of previously undiagnosed individuals with $\mathrm{TB}$ were symptomatic, of whom $50 \%$ had sought care for their illness at a health facility. ${ }^{12}$ Furthermore, once patients do access healthcare services, their TB illness may be missed-this has been shown to be a common problem in recent standardised patient studies conducted in Kenya, ${ }^{37}$ India $^{38}$ and China. ${ }^{39}$

Collectively, this suggests that both community-based and facility-based active TB case finding strategies, as well as training of healthcare providers to improve recognition of and testing for TB, are likely to be important activities to increase detection of individuals with $\mathrm{TB}$ in Zambia. Community-based active TB case finding may help overcome individuals' barriers to health-seeking and accessing TB services, possibly resulting in a greater absolute number of patients with TB diagnosed and patients who are detected earlier. ${ }^{40-42}$ However, effective 
and sustainable community-based active TB case finding strategies are not well described and represent an urgent TB research need. ${ }^{3343}$ There is strong evidence demonstrating that facility-based, active TB case finding strategies are efficient and may yield a large number of cases that would otherwise have been missed, especially in high-burden settings. ${ }^{44-47}$ A recent study evaluating a multicomponent active TB case finding strategy in a highburden primary healthcare facility in Lusaka, Zambia found that total TB notifications increased by $35 \%$ during the intervention period; of the total TB cases, $91.5 \%$ were from facility-based case finding interventions, while $8.5 \%$ were from community-based case finding interventions. ${ }^{47}$ One important component of this strategy was the implementation of patient-friendly TB fast-track points at health facilities that improved access by allowing individuals with TB symptoms to skip the regular queue and undergo rapid screening and testing for TB. Further research is needed to understand what potential strategies to improve TB care engagement and diagnosis are most preferred by and acceptable to community members in high-burden settings.

We estimate that nearly $10 \%$ of individuals diagnosed with TB were LTFU prior to the initiation of TB treatment. Pretreatment LTFU is common in many high-burden settings, as demonstrated by a systematic review that found that 4\%-38\% (weighted proportion 18\%) of patients with TB in sub-Saharan Africa were lost at this step in the cascade. ${ }^{48}$ This may be accounted for by patients who died prior to initiation of therapy-a common finding among such patients—and patients who cannot be traced after diagnosis either due to missing/incorrect contact information or because they have moved away. A recent qualitative study among patients with $\mathrm{TB}$ and healthcare workers (HCW) in India provided further understanding of the factors that may contribute to LTFU prior to initiation of TB therapy. ${ }^{49}$ The authors identified challenges and constraints related to organisational and administrative barriers resulting in patient disengagement from TB services over frustration, as well as negative HCW attitudes and behaviours resulting in patient distrust and feeling that their autonomy had been violated. There is an important need to design, evaluate and implement strategies that may address patient-level and health systems factors and reduce pretreatment LTFU. ${ }^{48}$ It should be noted that pretreatment loss-to-follow-up estimates may be overestimated because they fail to account for individuals who were in fact started on TB therapy but were not officially registered and therefore never notified to the NTP (under-notification). Zambia's NTP has recently completed a study to estimate the proportion of patients who are diagnosed but not notified, as well as the proportion of those who are started on treatment but never reported. This study will yield improved estimates of pretreatment loss-to-follow-up, which will allow for improved evaluations of programmatic changes that aim to improve TB diagnosis and linkage to TB treatment and care.
We found that important progress has been made in Zambia with regard to microbiological TB diagnosis and TB treatment completion from 2015 to 2018. During this period there was a massive effort to scale up the availability of Xpert MTB/RIF as the first-line TB diagnostic for all forms of TB. This was associated with a $12 \%$ increase in the proportion of patients with $\mathrm{TB}$ who were microbiologically confirmed (2692 additional annual DS-TB patients identified). Importantly, because Xpert also provides rapid simultaneous detection of rifampicin resistance, its scale-up was also associated with a threefold increase in patients with RR-TB detected and a fivefold increase in the number of patients with RR-TB who were notified and started on TB treatment. Zambia is currently preparing to scale up Xpert Ultra cartridges, which when paired with continued efforts to decentralise Xpert testing should allow for further gains in the detection of HIV-associated TB, extrapulmonary TB and RR-TB. ${ }^{50}$ There was also evidence of improved $\mathrm{TB}$ treatment completion rates for nearly all forms of TB between 2015 and 2018. While it is important to recognise progress that has been made, smaller but critically important gaps in the TB care cascade remain due to missed diagnoses and lack of treatment completion. Further efforts to expand access to microbiological TB testing and interventions to bolster TB treatment adherence that are grounded in person-centred care approaches, such as decentralisation of services coupled with improved education and communication as well as material and psychological support, are needed. ${ }^{51} 52$

PLHIV accounted for $60 \%$ of DS-TB cases in Zambia and were more likely to be lost at several steps of the cascade compared with HIV-negative individuals. This finding emphasises the need to strengthen HIV-TB collaborative activities. ${ }^{33} 53$ Due to non-specific clinical presentations and radiographic findings, one of the most important challenges to improving HIV-associated TB outcomes remains TB diagnosis. ${ }^{54}$ Non-specific symptoms may delay care-seeking among PLHIV, and without systematic TB screening among PLHIV presenting to and in care the diagnosis of many $\mathrm{TB}$ cases may be further delayed or missed. Systematic screening for TB at each clinical presentation ${ }^{55}$ must be coupled with access to improved microbiological diagnostic tools such as Xpert Ultra ${ }^{56}$ and urine lipoarabinomannan (LAM) ${ }^{56} 57$ testing to facilitate rapid $\mathrm{TB}$ detection and $\mathrm{TB}$ treatment initiation in order to minimise pretreatment LTFU and improve clinical outcomes. Compared with HIV-negative patients, HIVpositive patients were less likely to complete TB therapy, and TB treatment completion rates among PLHIV did not significantly change over a 4-year period from 2015 to 2018. Previously, a study among PLHIV in Zambia found that a large number of individuals LTFU from HIV services had died and that programmatic mortality rates were substantially under-reported; ${ }^{23}$ this suggests that mortality among PLHIV LTFU from TB treatment services is high and that TB-related mortality among PLHIV in Zambia is likely underestimated. The implementation of tailored 
interventions to improve adherence to TB treatment ${ }^{5158}$ as well as ${ }^{59} \mathrm{ART}$ among this highly vulnerable population therapy is needed.

Notably, we found that less than a quarter of RR-TB cases in 2018 were detected, started on appropriate treatment and completed appropriate therapy. This was despite improved access to rapid drug susceptibility via the scale-up of Xpert MTB/RIF testing from 2015 to 2018 and shorter and simplified drug-resistant $\mathrm{TB}$ regimens being introduced in 2018. ${ }^{16}$ The high rate of attrition of patients with RR-TB throughout the care cascade argues for the need for specific investments in systems strengthening to improve drug-resistant TB diagnosis and treatment in Zambia, mirroring this dire need in most high TB burden countries. ${ }^{19336061}$ One important contributing factor to the large number of patients with RR-TB not accessing DST is the high proportion of patients who are being diagnosed clinically and/or on the basis of radiological findings only-this accounted for approximately $44 \%$ of pulmonary TB cases in Zambia in 2018. Notably, the scale-up of Xpert testing between 2015 and 2018 was associated with more than $30 \%$ reduction in the proportion of RR-TB/MDR-TB cases that were LTFU after diagnosis and prior to initiation of treatment. This is likely due to the substantially faster detection of rifampicin resistance compared with conventional culture-based methods. Collectively, this demonstrates the importance of continued efforts to expand access to Xpert testing in Zambia in order to facilitate confirmation of TB diagnoses coupled with rapid detection of rifampicin resistance. While the implementation of existing diagnostic tools as well as improved DR-TB treatment regimens must be optimised, there remains a continued need for the development of rapid low-cost DST that can be scaled up to provide decentralised access to first-line and secondline DST aligned with current treatment recommendations, ${ }^{62}$ as well as continued progress towards shorter, less toxic and more effective DR-TB treatment regimens. ${ }^{63}$

This study used a validated analysis $\operatorname{method}^{6}$ incorporating a number of data sources to derive nationally representative estimates of the TB care cascade in Zambia; however, there were some limitations. As with other published TB cascades analyses, there is uncertainty around the estimates, especially the overall number of TB cases. The total burden of TB was calculated using indirect estimates from modelling that were based on case notification data and a prior national TB prevalence survey. We derived a conservative estimate of the total TB burden that accounted for missed cases from the prior year ${ }^{8}$ and that therefore may be a more appropriate estimate than measurements of TB incidence, which are rarely feasible to directly estimate. ${ }^{64}$ Due to a lack of a unique national patient identifier, we were unable to link specific individuals with their outcomes as they progressed through the TB care cascade, and thus unique individuals in one step of the cascade may differ from those in the following step; where possible, we attempted to account for duplicate diagnostic and treatment data, which were uncommon.
Implementation of a unique TB patient identifier and an improved TB data surveillance programme with enhanced data integration would greatly improve future estimates and allow for real-time individual-level, facilitylevel and subnational-level data to inform programme strengthening.

Given the potential importance of gender to TB epidemiology ${ }^{32}$ and potential differential health-seeking behaviours and access to TB services, ${ }^{36} 6667$ we sought to characterise the TB care cascade among men and women. For example, the prevalence of TB among men in Zambia's first national TB prevalence survey in 2013/2014 was almost twice as high as that among women (833 vs 487 cases per 100000 persons), ${ }^{12}$ and men with presumptive TB were less likely to have sought care for their symptoms than women $(31.4 \%$ vs $38.4 \%) .{ }^{68}$ Unfortunately, sex-disaggregated data sources were not available that would have allowed for each step of the cascade to be estimated. It is important that TB programmes collect sex-disaggregated diagnostic and treatment data to help ensure equity in access and treatment benefits. Additionally, because incidence, diagnosis, notification and treatment numbers are from 2018, we feel our analysis accurately represents the national TB care cascade in 2018; however, pretreatment LTFU estimates were informed by patient-level data from 2017 and the proportion of cases with rifampicin resistance was informed by higher-end estimates from the most recent national drug resistance survey conducted in 2008. ${ }^{22}$ An updated drug resistance survey is currently underway and will provide new estimates that will better guide programmatic priorities. Finally, to our knowledge, there are no locally or regionally representative estimates of $\mathrm{TB}$ relapse rates after documented TB treatment completion. This is an important quality metric of individuals' adherence to therapy as well as TB treatment programmes and should be assessed in future research studies. ${ }^{6}$

In conclusion, in 2018 only $45 \%$ of individuals with TB in Zambia completed the TB care cascade, and most losses were among patients who never accessed TB testing. Additionally, only $22 \%$ of all patients with RR-TB successfully completed appropriate TB treatment, and HIV-positive patients had substantially worse TB outcomes compared with HIV-negative patients. Our results suggest that continued systems strengthening coupled with patientcentred engagement strategies is required throughout the TB cascade of care; however, implementation of active TB case finding strategies, coupled with a renewed focus on those with rifampicin resistance and PLHIV, is urgently needed to improve TB-related outcomes and TB control in Zambia.

\section{Author affiliations}

${ }^{1}$ National Tuberculosis and Leprosy Control Programme, Lusaka, Zambia ${ }^{2}$ Department of Internal Medicine, University Teaching Hospital, Lusaka, Zambia ${ }^{3}$ Division of HIV, Infectious Diseases and Global Medicine, Zuckerberg San Francisco General Hospital and Trauma Center, University of California San Francisco, San Francisco, California, USA

${ }^{4}$ Department of Public Health and Research, Ministry of Health, Lusaka, Zambia 
${ }^{5}$ Tuberculosis Department, Center for Infectious Disease Research in Zambia, Lusaka, Zambia

${ }^{6}$ Department of Public Health and Community Medicine, Tufts University School of Medicine, Boston, Massachusetts, USA

${ }^{7}$ Ministry of Health, Lusaka, Zambia

Contributors PL, ADK and MM conceived the study. PL, RC, AS and KM were responsible for project administration. CCK, JM and SN collected and organised the data. ADK conducted the analysis and developed the figures, with input from PL, MM, RS, MK, CCK, JM, SN, RC, AS and KM. ADK, PL and MM wrote the first draft of the manuscript. All authors contributed to interpretation of data and editing of the article and approved the final version of the manuscript before submission.

Funding ADK was supported by the National Institute of Allergy and Infectious Diseases (Grant No T32 Al060530). This work was assisted in part by a CFAR-ARI Boost Award from the UCSF-Gladstone Center for AIDS Research (NIH P30Al027763).

Disclaimer The funding sources had no role in the study design, in the collection, analysis and interpretation of data, in the writing of the report, or in the decision to submit the manuscript for publication.

Competing interests None declared.

Patient consent for publication Not required.

Ethics approval Because this was a retrospective, population-level analysis without the use of any patient identifiers, the University of Zambia Biomedical Research Ethics Committee determined that this study met the criteria for exempt status (REF 001-02-21).

Provenance and peer review Not commissioned; externally peer reviewed.

Data availability statement All data relevant to the study are included in the article or uploaded as supplementary information.

Supplemental material This content has been supplied by the author(s). It has not been vetted by BMJ Publishing Group Limited (BMJ) and may not have been peer-reviewed. Any opinions or recommendations discussed are solely those of the author(s) and are not endorsed by BMJ. BMJ disclaims all liability and responsibility arising from any reliance placed on the content. Where the content includes any translated material, BMJ does not warrant the accuracy and reliability of the translations (including but not limited to local regulations, clinical guidelines, terminology, drug names and drug dosages), and is not responsible for any error and/or omissions arising from translation and adaptation or otherwise.

Open access This is an open access article distributed in accordance with the Creative Commons Attribution Non Commercial (CC BY-NC 4.0) license, which permits others to distribute, remix, adapt, build upon this work non-commercially, and license their derivative works on different terms, provided the original work is properly cited, appropriate credit is given, any changes made indicated, and the use is non-commercial. See: http://creativecommons.org/licenses/by-nc/4.0/.

\section{ORCID iDs}

Patrick Lungu http://orcid.org/0000-0002-6349-1885

Andrew D Kerkhoff http://orcid.org/0000-0002-5023-6658

Ramnath Subbaraman http://orcid.org/0000-0002-2063-943X

\section{REFERENCES}

1 World Health Organization. The End TB strategy. Geneva: World Health Organization, 2015

2 World Health Organization. Global tuberculosis report 2020. Geneva: World Health Organization, 2020.

3 Centers for Disease Control. CDC in Zambia. Center for disease control and prevention. Atlanta, 2017.

4 Medland NA, McMahon JH, Chow EPF, et al. The HIV care cascade: a systematic review of data sources, methodology and comparability. $J$ Int AIDS Soc 2015:18:20634.

5 Reid MJA, Goosby E. Lessons learned from the HIV care cascade can help end TB. Int J Tuberc Lung Dis 2017;21:245-6.

6 Subbaraman R, Nathavitharana RR, Mayer KH, et al. Constructing care cascades for active tuberculosis: a strategy for program monitoring and identifying gaps in quality of care. PLoS Med 2019;16:e1002754.

7 Subbaraman R, Nathavitharana RR, Satyanarayana S, et al. The tuberculosis cascade of care in India's public sector: a systematic review and meta-analysis. PLoS Med 2016;13:e1002149-38.
8 Naidoo P, Theron G, Rangaka MX, et al. The South African tuberculosis care cascade: estimated losses and methodological challenges. J Infect Dis 2017;216:S702-13.

9 Knoblauch AM, Grandjean Lapierre S, Randriamanana D, et al. Multidrug-resistant tuberculosis surveillance and cascade of care in Madagascar: a five-year (2012-2017) retrospective study. BMC Med 2020;18:173.

10 United Nations Population Fund. Zambia - Overview. Geneva: United Nations Population Fund, 2020.

11 Joint United Nations Programme on HIV/AIDS. Country factsheets - Zambia 2019. Joint United Nations Programme on HIV/AIDS. Geneva, 2020.

12 Kapata N, Chanda-Kapata P, Ngosa W, et al. The prevalence of tuberculosis in Zambia: results from the first national TB prevalence survey, 2013-2014. PLoS One 2016;11:e0146392.

13 Zambian Ministry of Health. Underreporting of TB patients in Zambia. National TB and leprosy programme, Lusaka. 2019

14 The National Tuberculosis and Leprosy Program. Guidelines for the programmatic management of drug-resistant tuberculosis in Zambia. 2017.

15 World Health Organization. Guidelines for treatment of drugsusceptible tuberculosis and patient care. Geneva: World Health Organization, 2017.

16 Challenge TB. Rapid scale-up of new drugs and regimens for the treatment of drug-resistant TB in Zambia. Zambia Office, Lusaka, 2020.

17 Zambia Ministry of Health. Zambia consolidated guidelines for treatment and prevention of HIV infection, 2018.

18 World Health Organization. Global tuberculosis report 2018. Geneva: World Health Organization, 2018.

19 World Health Organization. Global tuberculosis report 2019. Geneva: World Health Organization, 2019.

20 Glaziou P, Sismanidis C, Dodd PJ. Methods used by WHO to estimate the global burden of TB disease. Geneva: World Health Organization, 2018.

21 World Health Organization. Tuberculosis data. Available: https://www. who.int/teams/global-tuberculosis-programme/data

22 Kapata N, Mbulo G, Cobelens F, et al. The second Zambian national tuberculosis drug resistance survey - a comparison of conventional and molecular methods. Trop Med Int Health 2015;20:1492-500.

23 Holmes CB, Sikazwe I, Sikombe K, et al. Estimated mortality on HIV treatment among active patients and patients lost to follow-up in 4 provinces of Zambia: findings from a multistage sampling-based survey. PLoS Med 2018;15:e1002489.

24 Zambia Statistics Agency, Ministry of Health (MOH) Zambia, and ICF. Zambia demographic and health survey 2018. Lusaka, Zambia, and Rockville, Maryland, USA: Zambia Statistics Agency, Ministry of Health, and ICF, 2019

25 Horne DJ, Kohli M, Zifodya JS, et al. Xpert MTB/RIF and Xpert MTB/ RIF ultra for pulmonary tuberculosis and rifampicin resistance in adults. Cochrane Database Syst Rev 2019;6:CD009593.

26 Boehme CC, Nicol MP, Nabeta P, et al. Feasibility, diagnostic accuracy, and effectiveness of decentralised use of the Xpert MTB/ RIF test for diagnosis of tuberculosis and multidrug resistance: a multicentre implementation study. Lancet 2011;377:1495-505.

27 Steingart KR, Henry M, Ng V, et al. Fluorescence versus conventional sputum smear microscopy for tuberculosis: a systematic review. Lancet Infect Dis 2006;6:570-81.

28 World Health Organization. The use of molecular line probe assays for the detection of resistance to isoniazid and rifampicin. Geneva: World Health Organization, 2016.

29 Tortoli E, Benedetti M, Fontanelli A, et al. Evaluation of automated BACTEC MGIT 960 system for testing susceptibility of Mycobacterium tuberculosis to four major antituberculous drugs: comparison with the radiometric BACTEC 460TB method and the agar plate method of proportion. J Clin Microbiol 2002;40:607-10.

30 World Health Organization. Guidance for national tuberculosis programmes on the management of tuberculosis in children. Genevea: World Health Organization, 2014.

31 Dye C. Breaking a law: tuberculosis disobeys Styblo's rule. Bull World Health Organ 2008;86:4.

32 Horton KC, MacPherson P, Houben RMGJ, et al. Sex differences in tuberculosis burden and notifications in low- and middle-income countries: a systematic review and meta-analysis. PLoS Med 2016;13:e1002119.

33 Reid MJA, Arinaminpathy N, Bloom A, et al. Building a tuberculosisfree world: the Lancet Commission on tuberculosis. Lancet 2019;393:1331-84.

34 Subbaraman R, Jhaveri T, Nathavitharana RR. Closing gaps in the tuberculosis care cascade: an action-oriented research agenda. $J$ Clin Tuberc Other Mycobact Dis 2020;19:100144. 
35 de Vries SG, Cremers AL, Heuvelings CC, et al. Barriers and facilitators to the uptake of tuberculosis diagnostic and treatment services by hard-to-reach populations in countries of low and medium tuberculosis incidence: a systematic review of qualitative literature. Lancet Infect Dis 2017;17:e128-43.

36 Krishnan L, Akande T, Shankar AV, et al. Gender-related barriers and delays in accessing tuberculosis diagnostic and treatment services: a systematic review of qualitative studies. Tuberc Res Treat 2014;2014:1-14.

37 Daniels B, Dolinger A, Bedoya G, et al. Use of standardised patients to assess quality of healthcare in Nairobi, Kenya: a pilot, crosssectional study with international comparisons. BMJ Glob Health 2017;2:e000333.

38 Kwan A, Daniels B, Saria V, et al. Variations in the quality of tuberculosis care in urban India: a cross-sectional, standardized patient study in two cities. PLoS Med 2018;15:e1002653.

39 Sylvia S, Xue H, Zhou C, et al. Tuberculosis detection and the challenges of integrated care in rural China: a cross-sectional standardized patient study. PLoS Med 2017;14:e1002405.

40 Kranzer K, Afnan-Holmes H, Tomlin K, et al. The benefits to communities and individuals of screening for active tuberculosis disease: a systematic review. Int J Tuberc Lung Dis 2013;17:432-46.

41 STOB TB Partnership. Finding the missing people with TB in communities. Geneva: Stop TB Partnership, 2018.

42 Marks GB, Nguyen NV, Nguyen PTB, et al. Community-wide screening for tuberculosis in a high-prevalence setting. New Engl J Med 2019;381:1347-57.

43 Kerkhoff AD, Muyoyeta M, Cattamanchi A. Community-wide screening for tuberculosis. New Engl J Med 2020;382:1185-6.

44 Shapiro AE, Chakravorty R, Akande T, et al. A systematic review of the number needed to screen to detect a case of active tuberculosis in different risk groups. Geneva: World Health Organization, 2013.

45 STOB TB Partnership. Intensified TB case finding at facility level. Geneva: Stop TB Partnership, 2018.

46 Hanrahan CF, Nonyane BAS, Mmolawa L, et al. Contact tracing versus facility-based screening for active TB case finding in rura South Africa: a pragmatic cluster-randomized trial (Kharitode TB). PLoS Med 2019;16:e1002796.

47 Kagujie M, Chilukutu L, Somwe P, et al. Active TB case finding in a high burden setting; comparison of community and facility-based strategies in Lusaka, Zambia. PLoS One 2020;15:e0237931.

48 MacPherson P, Houben RMGJ, Glynn JR, et al. Pre-treatment loss to follow-up in tuberculosis patients in low- and lower-middle-income countries and high-burden countries: a systematic review and metaanalysis. Bull World Health Organ 2014;92:126-38.

49 Thomas BE, Suresh C, Lavanya J, et al. Understanding pretreatment loss to follow-up of tuberculosis patients: an explanatory qualitative study in Chennai, India. BMJ Glob Health 2020;5:e001974.

50 Dorman SE, Schumacher SG, Alland D, et al. Xpert MTB/RIF ultra for detection of Mycobacterium tuberculosis and rifampicin resistance: a prospective multicentre diagnostic accuracy study. Lancet Infect Dis 2018;18:76-84.

51 Alipanah N, Jarlsberg L, Miller C, et al. Adherence interventions and outcomes of tuberculosis treatment: a systematic review and meta-analysis of trials and observational studies. PLoS Med 2018;15:e1002595.

52 World Health Organization. A patient-centered approach to TB care. Geneva: World Health Organization, 2018.

53 World Health Organization. WHO policy on collaborative TB/ HIV activities: guidelines for national programmes and other stakeholders. Geneva: World Health Organization, 2012.

54 Kerkhoff AD, Cattamanchi A. Diagnosis of HIV-associated tuberculosis. In: Sereti I, Bisson G, Meintjes G, eds. HIV and tuberculosis: a formidable alliance. Cham: Springer, 2019: 127-59.

55 World Health Organization. WHO consolidated guidelines on tuberculosis, module 2: systematic screening for tuberculosis disease. Geneva: World Health Organization, 2021.

56 World Health Organization. WHO meeting report of a technical expert consultation: non-inferiority analysis of Xpert MTB/RIF ultra compared to Xpert MTB/RIF. Geneva: World Health Organization, 2017.

57 World Health Organization. Lateral flow urine lipoarabinomannan assay (LF-LAM) for the diagnosis of active tuberculosis in people living with HIV. Geneva: World Health Organization, 2019.

58 Subbaraman R, de Mondesert L, Musiimenta A, et al. Digital adherence technologies for the management of tuberculosis therapy: mapping the landscape and research priorities. BMJ Glob Health 2018;3:e001018.

59 Kanters S, Park JJH, Chan K, et al. Interventions to improve adherence to antiretroviral therapy: a systematic review and network meta-analysis. Lancet HIV 2017;4:e31:40-e40.

60 Dowdy DW, Theron G, Tornheim JA, et al. Drug-resistant tuberculosis in 2017: at a crossroads. Lancet Respir Med 2017;5:241-2.

61 Dheda K, Gumbo T, Maartens G, et al. The epidemiology, pathogenesis, transmission, diagnosis, and management of multidrug-resistant, extensively drug-resistant, and incurable tuberculosis. Lancet Respir Med 2017;5:291-360.

62 World Health Organization. High-priority target product profiles for new tuberculosis diagnostics: report of a consensus meeting. Geneva: World Health Organization, 2014.

63 Kendall EA, Sahu S, Pai M, et al. What will it take to eliminate drugresistant tuberculosis? Int J Tuberc Lung Dis 2019;23:535-46.

64 Stop TB Partnership. TB impact measurement. Geneva: Stop TB Partnership, 2012.

65 Horton KC, Sumner T, Houben RMGJ, et al. A bayesian approach to understanding sex differences in tuberculosis disease burden. Am J Epidemiol 2018;187:2431-8.

66 Chikovore J, Hart G, Kumwenda M, et al. 'For a mere cough, men must just CheW Conjex, gain strength, and continue working': the provider construction and tuberculosis care-seeking implications in Blantyre, Malawi. Glob Health Action 2015;8:26292.

67 Chikovore J, Hart G, Kumwenda M, et al. TB and HIV stigma compounded by threatened masculinity: implications for TB health-care seeking in Malawi. Int $J$ Tuberc Lung Dis 2017;21:26-33.

68 Chanda-Kapata P, Kapata N, Masiye F, et al. Health seeking behaviour among individuals with presumptive tuberculosis in Zambia. PLoS One 2016;11:e0163975. 